\title{
Strong and Confined Acids Enable a Catalytic Asymmetric Nazarov Cyclization of Simple Divinyl Ketones
}

\author{
Jie Ouyang, Jennifer L. Kennemur, Chandra Kanta De, Christophe Farès, ${ }^{\bullet}$ and Benjamin List*๑ \\ Max-Planck-Institut für Kohlenforschung, Kaiser-Wilhelm-Platz 1, 45470 Mülheim an der Ruhr, Germany \\ Supporting Information
}

ABSTRACT: We report a catalytic asymmetric Nazarov cyclization of simple, acylic, alkyl-substituted divinyl ketones using our recently disclosed strong and confined imidodiphosphorimidate Brønsted acids. The corresponding monocyclic cyclopentenones are formed in good yields and excellent regio-, diastereo-, and enantioselectivities. Further, the chemical utility of the obtained enantiopure cyclopentenones is demonstrated.

$\mathrm{E}$ nantiopure cyclopentenones are frequently used as key building blocks toward, and are themselves present within, a variety of bioactive and/or complex natural products. ${ }^{1}$ Chemists have consequently devoted considerable effort to the development of enantioselective approaches to these important compounds. Commonly used techniques today include chemical or enzymatic resolutions, ${ }^{2,3}$ asymmetric functionalizations of existing cyclopentenone units, ${ }^{1 \mathrm{c}}$ or derivatizations of chiral-pool reagents. ${ }^{4}$ While effective, each of these strategies is conceptually inferior to synthetic methods that introduce chirality during the construction of the cyclic unit from simple starting materials, such as asymmetric Pauson-Khand reactions or Nazarov cyclizations. ${ }^{5,6}$ Unfortunately, the relatively underdeveloped methodology of the latter techniques has limited their application. In fact, despite being considered one of the most direct and atom-economical transformations for the synthesis of cyclopentenones, the asymmetric Nazarov cyclization is arguably one of the least employed methods toward chiral cyclopentenones. ${ }^{7}$ The limited application of this strategy is likely an effect of systematic substrate specificity for given variants and, therefore, a lack of generality.

Since the first catalytic asymmetric Nazarov cyclization emerged from the Trauner group in $2003,{ }^{7 \mathrm{c}}$ this and subsequent methods have largely depended on designed substrates to overcome the relatively low reactivity of divinyl ketones and/or to circumvent challenges in regio- and stereoselectivity (Figure 1a). More specifically, these substrates are usually activated by adjacent heteroatoms to stabilize the oxyallyl cation, neighboring electron-withdrawing groups, and/ or $\beta$-aryl substituents to polarize the divinyl ketone. ${ }^{6 \mathrm{~b}}$ Notably, in 2013, Rawal and co-workers disclosed two Nazarov cyclizations of electronically unactivated divinyl ketones; however, in each of these substrates, one of the olefins was within a cyclohexane unit, compromising the overall generality of the method. ${ }^{7 j}$ As such, we recognized that simple alkylsubstituted, acyclic divinyl ketones still remain an extremely

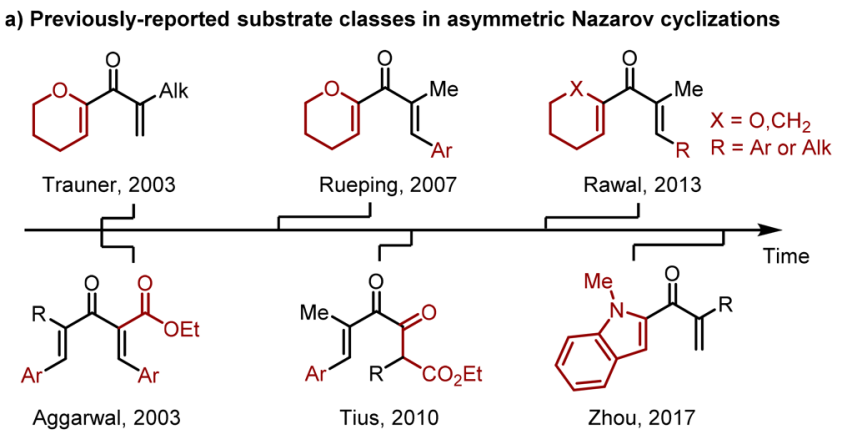

b) This work: asymmetric Nazarov cyclizations of simple divinyl ketones

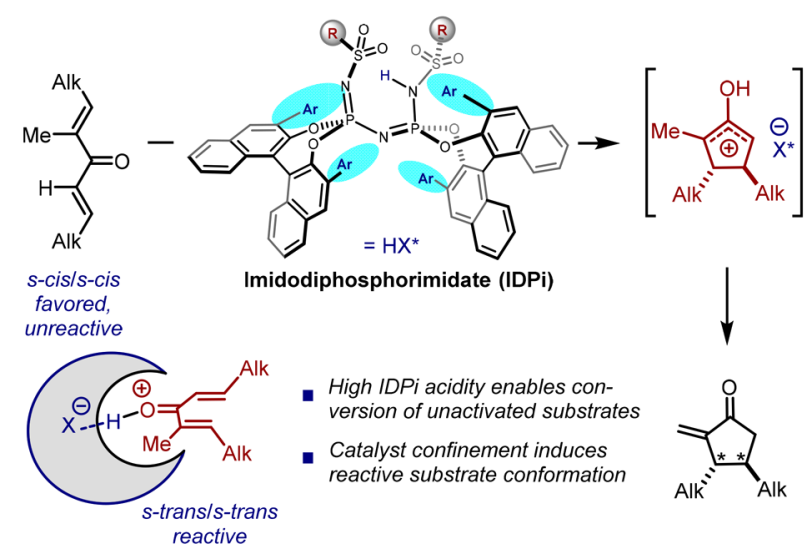

Figure 1. (a) Previously reported systems for asymmetric Nazarov cyclizations. (b) Highly acidic and confined acid enables catalytic asymmetric Nazarov cyclization of simple divinyl ketones.

challenging class of substrates for asymmetric Nazarov cyclizations and thereby undermine its synthetic application.

Recently, our group disclosed a novel class of chiral, highly acidic, and confined Brønsted acids, i.e., imidodiphosphorimidates (IDPis), and demonstrated their success in a variety of asymmetric transformations. ${ }^{8}$ We envisioned that these highly reactive catalysts might be uniquely suited for the Nazarov cyclization of unbiased divinyl ketones, as the confined chiral microenvironment not only induces asymmetry but furthermore may enhance reactivity by increasing the population of the reactive $s$-trans/s-trans conformer of the divinyl ketone (Figure 1b). Here, we report the fruition of these concepts with a unique catalytic asymmetric Nazarov

Received: December 31, 2018

Published: February 15, 2019 
cyclization of simple, acylic, and alkyl-substituted divinyl ketones.

We initiated our studies by evaluating acyclic divinyl ketone 1a as a model substrate using a variety of chiral Brønsted acid catalysts in toluene at $25{ }^{\circ} \mathrm{C}$ (Scheme 1). As we anticipated,

Scheme 1. Reaction Development ${ }^{a}$

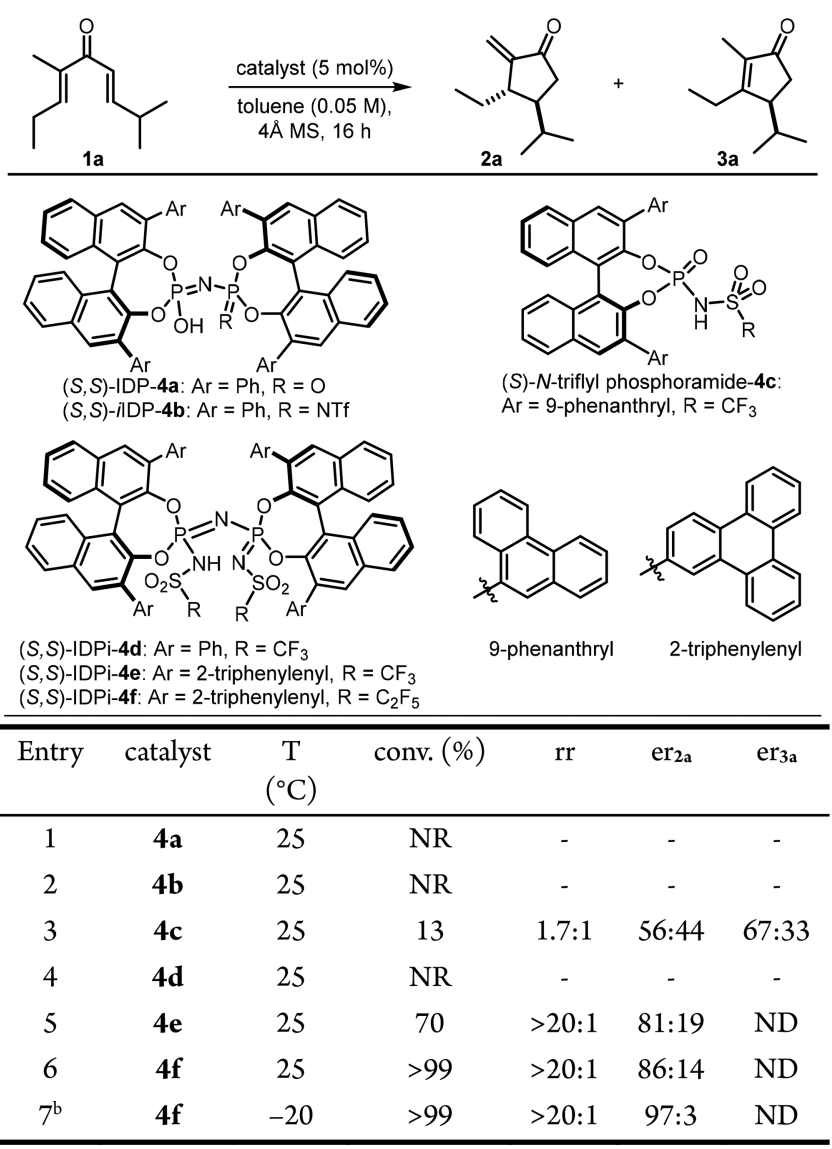

${ }^{a}$ Reactions were performed with substrate $\mathbf{1 a}(0.02 \mathrm{mmol})$, catalyst (5 $\mathrm{mol} \%), 4 \AA \mathrm{MS}(10 \mathrm{mg})$ in toluene $(0.4 \mathrm{~mL})$; conversions (conv) and regioisomeric ratios ( $\mathrm{rr}$ of $\mathbf{2 a}: 3 \mathrm{a}$ ) were obtained by ${ }^{1} \mathrm{H}$ NMR analysis with $\mathrm{Ph}_{3} \mathrm{CH}$ as an internal standard; enantiomeric ratios (er) were measured by GC, unless otherwise indicated; all diastereomeric ratios (dr) of product 2 a were $>20: 1 .{ }^{b}$ Reaction was run for 3.5 days. $\mathrm{NR}=$ no reaction; $\mathrm{ND}=$ not determined.

relatively weakly acidic and confined Brønsted acids, such as imidodiphosphoric acid (IDP) $\mathbf{4 a}$ and iminoimidodiphosphate (iIDP) $\mathbf{4 b}$, did not provide any of the desired products (Table 1 , entries 1 and 2). Interestingly, $N$-triflyl phosphoramide 4c, which Rueping and co-workers have already shown to be an efficient Brønsted acid of Nazarov cyclizations, resulted in poor conversion and regioselectivity $(2 \mathrm{a} / 3 \mathbf{a}=1.7: 1)$ and an enantiomeric ratio of $56: 44$ for $2 a$ and $67: 33$ for $3 a$ (entry 3 ).

Remarkably, even highly acidic IDPi catalyst 4d (where Ar = $\mathrm{Ph}$ ) proved to be inactive under the reaction conditions. However, based on our hypothesis that the confinement of the IDPi scaffold would be critical for the increased population of the necessary s-trans/s-trans conformer, we tested IDPi catalysts with sterically larger $\pi$-substituents in the $3,3^{\prime}$ positions. Indeed, upon testing IDPi catalysts $\mathbf{4 e}$ and $\mathbf{4 f}$ (where $\mathrm{Ar}=2$-triphenylenyl), $\mathbf{2 a}$ was formed in good yields with excellent diastereo- and regioselectivity (both $>20: 1$ ) and moderate enantioselectivity (entries 5 and 6). IDPi catalyst $4 \mathrm{f}$
Table 1. Scope of the Reaction ${ }^{a, b}$

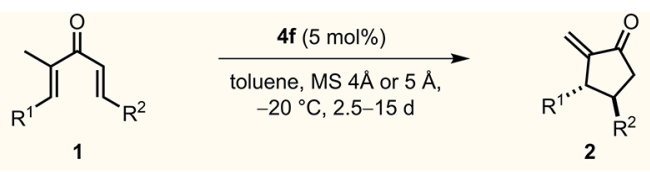

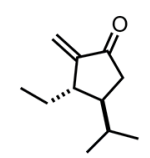

2a,7 d, $72 \%^{\mathrm{c}}$

2e, $14 \mathrm{~d}, 51 \%^{\mathrm{c}}$ 92:8 er<smiles>C=C1C(=O)CC(C2CCCCC2)[C@H]1CC</smiles>

2h, 4.5 d, $85 \%$ $>20: 1 \mathrm{rr}$ 96:4 er

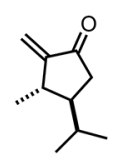

2I, $3.5 \mathrm{~d}, 66 \%^{\mathrm{c}}$ $>20: 1 \mathrm{rr}$ 95:5 er

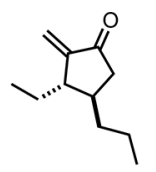

2b, 14 d, $62 \%^{c}$

$$
10: 1 \mathrm{rr}
$$$$
\text { 93:7 er }
$$<smiles>CCC1=C(C)C(=O)CC1C1CC1</smiles>

$3 e, 48 \%^{c}$ $>99: 1$ er

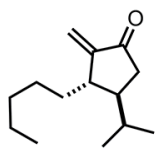

2i, 14 d, $72 \%$ $>20: 1 \mathrm{rr}$

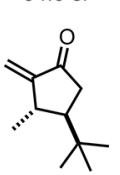

$2 m^{b}, 13 d, 72 \%^{c}$ $>20: 1$ rr 97:3 er<smiles>C=C1C(=O)CC(CC(C)C)[C@H]1CC</smiles>

2c, 13 d, $67 \%^{c}$ $>20: 1 \mathrm{rr}$ 93:7 er<smiles>C=C1C(=O)CC(C2CCC2)[C@H]1CC</smiles>

2f, $2.5 \mathrm{~d}, 64 \%^{\mathrm{c}}$ $>20: 1 \mathrm{rr}$ 96:4 er<smiles>C=C1C(=O)CC(C(C)C)[C@H]1CCCCCl</smiles>

2j, 14 d, $73 \%$ $>20: 1 \mathrm{rr}$

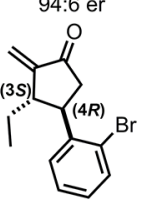

2n, 5 d, $71 \%$ $>20: 1 \mathrm{rr}$ 88:12 er

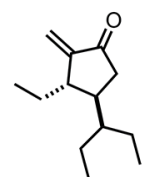

2d, 12 d, $77 \%$ $>20: 1 \mathrm{rr}$ 95:5 er<smiles>C=C1C(=O)CC(C2CCCC2)[C@@H]1CC</smiles>

$2 g, 4.5$ d, $78 \%$ $>20: 1 \mathrm{rr}$ $96: 4$ er<smiles>C=C1C(=O)CC(C(C)C)[C@H]1CCc1ccccc1</smiles>

2k, 15 d, $72 \%$ $12: 1 \mathrm{rr}$ 93:7 er
${ }^{a}$ Reactions were carried out with $0.2 \mathrm{mmol}$ of substrates $\mathbf{1}$, catalyst $4 \mathbf{f}$ $(5 \mathrm{~mol} \%)$, and $100 \mathrm{mg}$ molecular sieves in $4 \mathrm{~mL}$ of toluene $(0.05 \mathrm{M})$ at $-20{ }^{\circ} \mathrm{C}$ for the specified reaction time. Regioisomeric ratios ( $\mathrm{rr}$ of 2/3) and diastereomeric ratios ( $\mathrm{dr}$ ) were detected by ${ }^{1} \mathrm{H}$ NMR of the crude reaction mixture. All diastereomeric ratios $(\mathrm{dr})$ were $>20: 1$. The enantiomeric ratios (er) were determined by GC or HPLC analysis. ${ }^{b} 7 \mathrm{~mol} \%$ catalyst was used. ${ }^{c}$ Yields of the volatile products were determined by ${ }^{1} \mathrm{H}$ NMR analysis of the mixtures after column chromatography to remove toluene.

was found to be the best catalyst for this transformation in terms of enantioselectivity and was therefore selected for further optimizations. Gratifyingly, when the reaction was performed at $-20{ }^{\circ} \mathrm{C}$, full conversion of substrate 1a to enone 2a was observed with excellent regio- $(>20: 1)$, diastereo(>20:1), and enantioselectivity (97:3).

With the optimized conditions in hand, we next explored the scope of this reaction. ${ }^{9}$ Substituents at $\mathrm{R}^{2}$ with linear $(\mathbf{1 b})$, branched (1c, $\mathbf{1 d})$, and cyclic $(\mathbf{1} \mathbf{f}-\mathbf{h})$ aliphatic groups were well tolerated, providing the corresponding enones in good yields with excellent regio- and enantioselectivities. Interestingly, cyclopropyl-substituted substrate $1 \mathrm{e}$ resulted in two regioisomers, $2 \mathrm{e}$ and $3 \mathrm{e}(\mathrm{rr}=1: 1)$, under the reaction conditions. We suspect that the poor regioselectivity is a result of a relative increase in the thermodynamic stability of the endocyclic isomer $3 \mathbf{e}$ by virtue of the unique $\pi$-character of the cyclopropyl unit. The successful application of substrate $\mathbf{1 j}$, containing an alkyl chloride, potentially allows for subsequent 
cyclization or functionalization. In the case of substrate $\mathbf{1 k}$, a Friedel-Crafts-type interrupted Nazarov cyclization was not observed. ${ }^{10}$ We next turned our attention toward divinyl ketones $\mathbf{1 l}$ and $\mathbf{1 m}$ with a methyl substituent at $\mathrm{R}^{1}$. The desired enone 21 was obtained as a single regioisomer ( $\mathrm{rr}>20: 1)$ and with an excellent enantiomeric ratio of 95:5. As for the more bulky substituted divinyl ketone $1 \mathrm{~m}\left(\mathrm{R}^{2}=t\right.$-Bu group), a slightly higher catalyst loading $(7 \mathrm{~mol} \%)$ was required to give cyclopentanone $\mathbf{2 m}$ in good yield $(72 \%)$ and excellent enantioselectivity (97:3). Notably, o-bromophenyl divinyl ketone $1 \mathbf{n}$, as a representative of an aryl-substituted substrate, was converted with a reasonable er of $88: 12$. The absolute configuration of the produced ketone $\mathbf{2 n}$ was determined to be $3 S, 4 R$ following derivatization (see the SI). The relative configuration of all other products was assigned by analogy.

Encouraged by the success of our reaction design, we were eager to investigate the mechanism of this catalytic, asymmetric Nazarov cyclization. We envisioned two plausible scenarios, the first in which the free catalyst is the resting state and the second involving a covalent intermediate formed in a reaction between the oxyallyl cation and the anion of catalyst 4f, similar to that which was found in the imidodiphosphoric acid (IDP) catalyzed carbonyl-ene cyclization previously reported by our group. ${ }^{8 \mathrm{i}, 11}$ In order to distinguish these two possible mechanisms, a kinetic study was performed using ${ }^{1} \mathrm{H}$ NMR analysis. As shown in Figure 2a, the linear correlation

\section{a) Kinetic study ( ${ }^{1} \mathrm{H}$ NMR)}

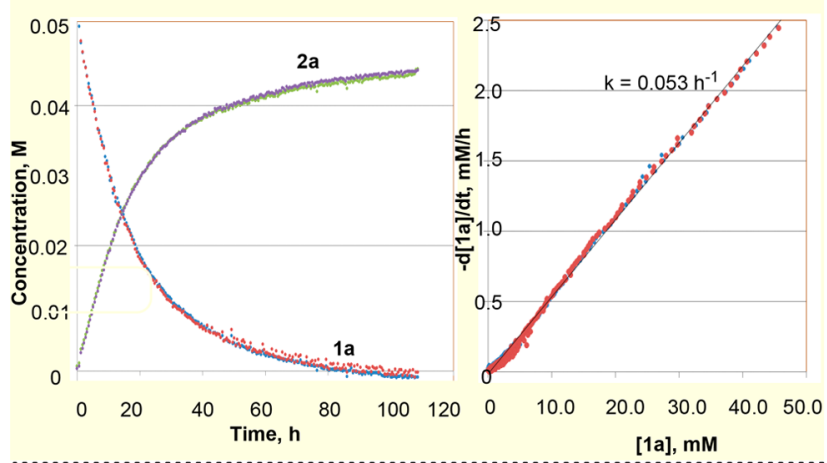

b) Proposed catalytic cycle

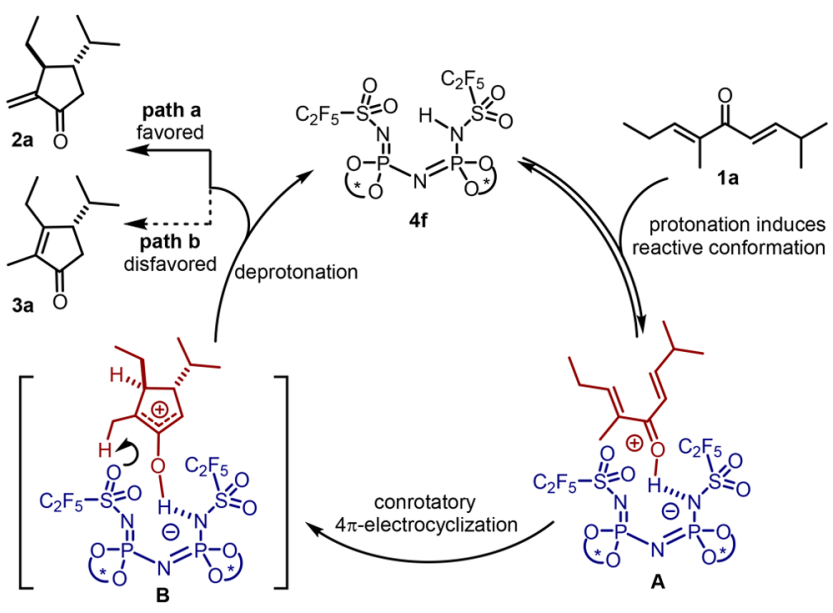

Figure 2. (a) Reaction profile for the reaction of substrate 1a with catalyst $4 \mathrm{f}$ in the presence of $4 \AA$ molecular sieves at $-20{ }^{\circ} \mathrm{C}$ in toluene- $d_{8}$ and $\mathrm{CH}_{2} \mathrm{Br}_{2}$ as external standard. (b) Proposed mechanism. between reaction rate and concentration of starting material suggests the reaction to be first order in substrate under the steady state approximation. We therefore propose that the free catalyst is the resting state in the catalytic cycle and coordinates to the substrate to form the complex A (Figure $2 b)$. Subsequently, a conrotatory $4 \pi$-electrocyclization occurs to generate the oxyallyl ion pair $\mathrm{B}$, followed by a kinetically controlled deprotonation (path a), presumably by the moderately basic $\mathrm{O}$ atoms of the sulfonyl group, which regenerates the catalyst and releases the product.

We also explored the synthetic utility of our enone products (Scheme 2). Indeed, unsaturated ketone $\mathbf{2 a}$ reacted as a

\section{Scheme 2. Functionalization of Nazarov Cyclization} Product 2a

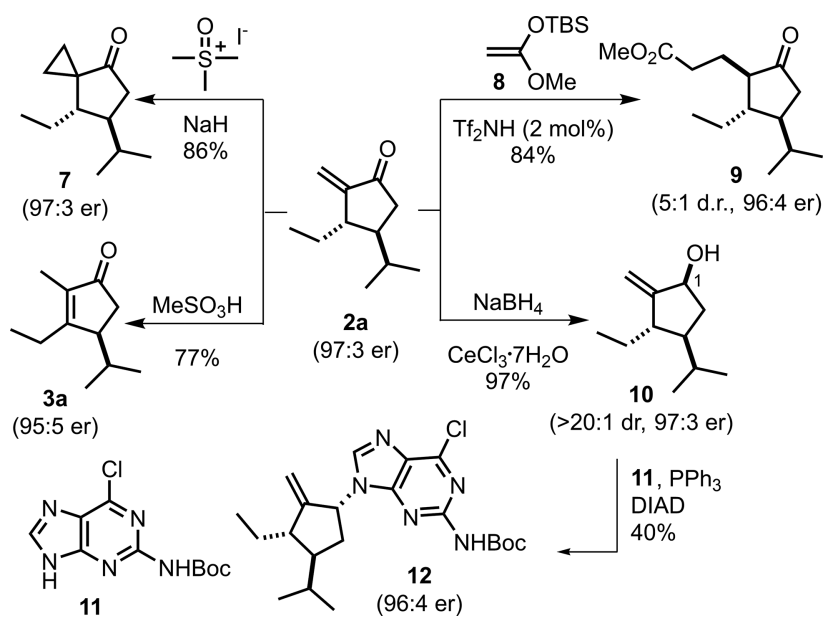

Michael acceptor in a cyclopropanation and in a MukaiyamaMichael addition. The resulting products, ketone 7 and cyclopentanone 9, were obtained without deterioration of enantioselectivity. The $\alpha$-methylene unit of 2 a could be isomerized to the fully substituted, thermodynamically more stable cyclopentenone $\mathbf{3 a}$ with an excess amount of methanesulfonic acid, again retaining the excellent enantioselectivity. Moreover, a Luche reduction of 2a furnished allylic alcohol 10 in excellent diastereoselectivity $(\mathrm{dr}>20: 1)$, which could then be utilized in a Mitsunobu reaction to install a purine-derivative and afford compound $\mathbf{1 2}$ with excellent $\mathrm{C} 1$ enantiopurity. ${ }^{12}$

In conclusion, we have developed a powerful catalytic, asymmetric Nazarov cyclization of simple, acyclic, aliphaticsubstituted divinyl ketones using a strong and confined Brønsted acid. We propose that the confinement of the IDPi scaffold induces the reactive s-trans/s-trans conformation of the divinyl ketone substrate, thereby promoting the cyclization to give a variety of versatile enones in good yields and excellent enantio-, regio-, and diastereoselectivities. Our approach could be useful in other conformation-dependent transformations, and the developed Nazarov reaction may aid in the asymmetric synthesis of several biologically active natural products.

\section{ASSOCIATED CONTENT}

S Supporting Information

The Supporting Information is available free of charge on the ACS Publications website at DOI: 10.1021 /jacs.8b13899.

Additional detailed synthetic protocols, analytical data for all compounds, and computational strategy (PDF) 


\section{AUTHOR INFORMATION}

\section{Corresponding Author}

*list@kofo.mpg.de

\section{ORCID $\odot$}

Christophe Farès: 0000-0001-6709-5057

Benjamin List: 0000-0002-9804-599X

\section{Notes}

The authors declare the following competing financial interest(s): A patent, WO2017037141 (A1), has been filed by the MPI für Kohlenforschung covering the IDPi catalyst class and their applications in asymmetric synthesis.

\section{ACKNOWLEDGMENTS}

Generous support from the Max Planck Society, the Deutsche Forschungsgemeinschaft (Leibniz Award to B.L), and the European Research Council (Advanced Grant " $\mathrm{C}-\mathrm{H}$ Acids for Organic Synthesis, CHAOS") is gratefully acknowledged. Excellent service provided by our technicians and our NMR, GC, and HPLC departments is gratefully acknowledged.

\section{REFERENCES}

(1) (a) Hanna, I. Synthesis of substituted furans using 1,4-dioxene. Tetrahedron Lett. 1999, 40, 2521-2524. (b) Gibson, S. E.; Lewis, S. E.; Mainolfi, N. Transition metal-mediated routes to cyclopentenones. J. Organomet. Chem. 2004, 689, 3873-3890. (c) Simeonov, S. P.; Nunes, J. P. M.; Guerra, K.; Kurteva, V. B.; Afonso, C. A. M. Synthesis of Chiral Cyclopentenones. Chem. Rev. 2016, 116, 5744-5893.

(2) Johnson, C. R.; Penning, T. D. Triply convergent synthesis of (-)-prostaglandin E2 methyl ester. J. Am. Chem. Soc. 1988, 110, $4726-4735$

(3) Pinot, E.; Guy, A.; Guyon, A.-L.; Rossi, J.-C.; Durand, T. Enzymatic kinetic resolution of a functionalized 4-hydroxy-cyclopentenone: synthesis of the key intermediates in the total synthesis of isoprostanes. Tetrahedron: Asymmetry 2005, 16, 1893-1895.

(4) Brill, Z. G.; Condakes, M. L.; Ting, C. P.; Maimone, T. J. Navigating the Chiral Pool in the Total Synthesis of Complex Terpene Natural Products. Chem. Rev. 2017, 117, 11753-11795.

(5) (a) Blanco-Urgoiti, J.; Añorbe, L.; Pérez-Serrano, L.; Domínguez, G.; Pérez-Castells, J. The Pauson-Khand reaction, a powerful synthetic tool for the synthesis of complex molecules. Chem. Soc. Rev. 2004, 33, 32-42. (b) Roche, S. P.; Aitken, D. J. Chemistry of 4-Hydroxy-2-cyclopentenone Derivatives. Eur. J. Org. Chem. 2010, 2010, 5339-5358.

(6) (a) Nazarov, I. N.; Zaretskaya, I. I. Izv. Akad. Nauk. SSSR, Ser. Khim. 1941, 211-224. (b) Frontier, A. J.; Collison, C. The Nazarov cyclization in organic synthesis. Recent advances. Tetrahedron 2005, 61, 7577-7606. (c) Pellissier, H. Recent developments in the Nazarov process. Tetrahedron 2005, 61, 6479-6517. (d) Tius, M. A. Some New Nazarov Chemistry. Eur. J. Org. Chem. 2005, 2005, 21932206. (e) Shimada, N.; Stewart, C.; Tius, M. A. Asymmetric Nazarov cyclizations. Tetrahedron 2011, 67, 5851-5870. (f) West, F. G.; Scadeng, O.; Wu, Y. K.; Fradette, R. J.; Joy, S. The Nazarov Cyclization. In Comprehensive Organic Synthesis II, 2nd ed.; Knochel, P., Ed.; Elsevier: Amsterdam, 2014; pp 827-866.

(7) (a) Aggarwal, V. K.; Belfield, A. J. Catalytic Asymmetric Nazarov Reactions Promoted by Chiral Lewis Acid Complexes. Org. Lett. 2003, 5, 5075-5078. (b) He, W.; Sun, X.; Frontier, A. J. Polarizing the Nazarov Cyclization: Efficient Catalysis under Mild Conditions. J. Am. Chem. Soc. 2003, 125, 14278-14279. (c) Liang, G.; Gradl, S. N.; Trauner, D. Efficient Nazarov Cyclizations of 2-Alkoxy-1,4-pentadien3-ones. Org. Lett. 2003, 5, 4931-4934. (d) Liang, G.; Trauner, D. Enantioselective Nazarov Reactions through Catalytic Asymmetric Proton Transfer. J. Am. Chem. Soc. 2004, 126, 9544-9545. (e) Rueping, M.; Ieawsuwan, W.; Antonchick, A. P.; Nachtsheim, B. J. Chiral Brønsted Acids in the Catalytic Asymmetric Nazarov Cyclization-The First Enantioselective Organocatalytic Electrocyclic
Reaction. Angew. Chem., Int. Ed. 2007, 46, 2097-2100. (f) Walz, I.; Togni, A. Ni(II)-catalyzed enantioselective Nazarov cyclizations. Chem. Commun. 2008, 4315-4317. (g) Basak, A. K.; Shimada, N.; Bow, W. F.; Vicic, D. A.; Tius, M. A. An Organocatalytic Asymmetric Nazarov Cyclization. J. Am. Chem. Soc. 2010, 132, 8266-8267. (h) Bow, W. F.; Basak, A. K.; Jolit, A.; Vicic, D. A.; Tius, M. A. Enamine-Iminium Ion Nazarov Cyclization of $\alpha$-Ketoenones. Org. Lett. 2010, 12, 440-443. (i) Raja, S.; Ieawsuwan, W.; Korotkov, V.; Rueping, M. Asymmetric Brønsted Acid-Catalyzed Nazarov Cyclization of Acyclic $\alpha$-Alkoxy Dienones. Chem. - Asian J. 2012, 7, 23612366. (j) Hutson, G. E.; Turkmen, Y. E.; Rawal, V. H. Salen promoted enantioselective Nazarov cyclizations of activated and unactivated dienones. J. Am. Chem. Soc. 2013, 135, 4988-4991. (k) Jolit, A.; Walleser, P. M.; Yap, G. P. A.; Tius, M. A. Catalytic Enantioselective Nazarov Cyclization: Construction of Vicinal All-Carbon-Atom Quaternary Stereocenters. Angew. Chem., Int. Ed. 2014, 53, 61806183. (1) Kitamura, K.; Shimada, N.; Stewart, C.; Atesin, A. C.; Ateşin, T. A.; Tius, M. A. Enantioselective Palladium(0)-Catalyzed Nazarov-Type Cyclization. Angew. Chem., Int. Ed. 2015, 54, 62886291. (m) Wang, G.-P.; Chen, M.-Q.; Zhu, S.-F.; Zhou, Q.-L. Enantioselective Nazarov cyclization of indole enones cooperatively catalyzed by Lewis acids and chiral Brønsted acids. Chem. Sci. 2017, 8, 7197-7202. (n) Jin, J.; Zhao, Y.; Gouranourimi, A.; Ariafard, A.; Chan, P. W. H. Chiral Brønsted Acid Catalyzed Enantioselective Dehydrative Nazarov-Type Electrocyclization of Aryl and 2-Thienyl Vinyl Alcohols. J. Am. Chem. Soc. 2018, 140, 5834-5841. (o) Mietke, T.; Cruchter, T.; Larionov, V. A.; Faber, T.; Harms, K.; Meggers, E. Asymmetric Nazarov Cyclizations Catalyzed by Chiral-at-Metal Complexes. Adv. Synth. Catal. 2018, 360, 2093-2100. (p) Süsse, L.; Vogler, M.; Mewald, M.; Kemper, B.; Irran, E.; Oestreich, M. Enantioselective Nazarov Cyclizations Catalyzed by an Axial Chiral C6F5-Substituted Boron Lewis Acid. Angew. Chem., Int. Ed. 2018, 57, 11441-11444.

(8) (a) Kaib, P. S.; Schreyer, L.; Lee, S.; Properzi, R.; List, B. Extremely Active Organocatalysts Enable a Highly Enantioselective Addition of Allyltrimethylsilane to Aldehydes. Angew. Chem., Int. Ed. 2016, 55, 13200-13203. (b) Xie, Y.; Cheng, G.-J.; Lee, S.; Kaib, P. S. J.; Thiel, W.; List, B. Catalytic Asymmetric Vinylogous Prins Cyclization: A Highly Diastereo- and Enantioselective Entry to Tetrahydrofurans. J. Am. Chem. Soc. 2016, 138, 14538-14541. (c) Lee, S.; Kaib, P. S. J.; List, B. Asymmetric Catalysis via Cyclic, Aliphatic Oxocarbenium Ions. J. Am. Chem. Soc. 2017, 139, 21562159. (d) Liu, L.; Kim, H.; Xie, Y.; Fares, C.; Kaib, P. S. J.; Goddard, R.; List, B. Catalytic Asymmetric [4+2]-Cycloaddition of Dienes with Aldehydes. J. Am. Chem. Soc. 2017, 139, 13656-13659. (e) Bae, H. Y.; Höfler, D.; Kaib, P. S. J.; Kasaplar, P.; De, C. K.; Döhring, A.; Lee, S.; Kaupmees, K.; Leito, I.; List, B. Approaching sub-ppm-level asymmetric organocatalysis of a highly challenging and scalable carbon-carbon bond forming reaction. Nat. Chem. 2018, 10, 888894. (f) Gatzenmeier, T.; Kaib, P. S. J.; Lingnau, J. B.; Goddard, R.; List, B. The Catalytic Asymmetric Mukaiyama-Michael Reaction of Silyl Ketene Acetals with $\alpha, \beta$-Unsaturated Methyl Esters. Angew. Chem., Int. Ed. 2018, 57, 2464-2468. (g) Gatzenmeier, T.; Turberg, M.; Yepes, D.; Xie, Y.; Neese, F.; Bistoni, G.; List, B. Scalable and Highly Diastereo- and Enantioselective Catalytic Diels-Alder Reaction of $\alpha, \beta$-Unsaturated Methyl Esters. J. Am. Chem. Soc. 2018, 140, 12671-12676. (h) Schreyer, L.; Kaib, P. S. J.; Wakchaure, V. N.; Obradors, C.; Properzi, R.; Lee, S.; List, B. Confined acids catalyze asymmetric single aldolizations of acetaldehyde enolates. Science 2018, 362, 216-219. (i) Tsuji, N.; Kennemur, J. L.; Buyck, T.; Lee, S.; Prévost, S.; Kaib, P. S. J.; Bykov, D.; Farès, C.; List, B. Activation of olefins via asymmetric Brønsted acid catalysis. Science 2018, 359, 1501-1505. (j) Lee, S.; Kaib, P. S.; List, B. N-Triflylphosphorimidoyl Trichloride: A Versatile Reagent for the Synthesis of Strong Chiral Brønsted Acids. Synlett 2017, 28, 1478-1480.

(9) A description of known limitations in the scope of the method is provided in the SI. 
(10) Bender, J. A.; Arif, A. M.; West, F. G. Nazarov-Initiated Diastereoselective Cascade Polycyclization of Aryltrienones. J. Am. Chem. Soc. 1999, 121, 7443-7444.

(11) Liu, L.; Leutzsch, M.; Zheng, Y.; Alachraf, M. W.; Thiel, W.; List, B. Confined Acid-Catalyzed Asymmetric Carbonyl-Ene Cyclization. J. Am. Chem. Soc. 2015, 137, 13268-13271.

(12) Xu, H.; Wang, F.; Xue, W.; Zheng, Y.; Wang, Q.; Qiu, F. G.; Jin, Y. Total Synthesis of Entecavir: A Robust Route for Pilot Production. Org. Process Res. Dev. 2018, 22, 377-384. 